\title{
CONCEPTUAL DESIGN OF A VACUUM SYSTEM FOR A COMPACT, HIGH LUMINOSITY CESR UPGRADE*
}

\author{
$\underline{\text { Kern W. Ormond }}^{\dagger}$, Joseph T. Rogers, Laboratory of Nuclear Studies, Cornell University
}

\begin{abstract}
A conceptual design study for a vacuum system for a possible compact high luminosity upgrade to CESR is presented. The vacuum chamber consists of an elliptical cross-section beam chamber connected to a pumping chamber by holes recessed in several channels in the beam chamber wall. Recessing the hole provides a decrease in the impedance of the beam chamber while still providing protection to the pumping chamber from $\mathrm{RF}$ fields generated by the beam. The beam chamber has a very compact cross-section compatible with twoin-one quadrupole magnets and inexpensive compact dipole magnets [1,2]. Pumping will be provided by a combination of non-evaporable getter (NEG) and ion pumps. Calculations were carried out of the impedance and loss factor of the chamber as well as transmission of $\mathrm{RF}$ field power through the slots and the conductance of the pumping slots. We have also calculated the linear synchrotron radiation power density and the pressure profile and beam-gas life time for this chamber and pump configuration. We consider the time between necessary NEG pump reactivations and the total capacity of the pumps.
\end{abstract}

\section{INTRODUCTION}

The vacuum system for a very high luminosity $\mathrm{e}^{+} \mathrm{e}^{-}$ collider must be capable of handling a large synchrotron radiation heat load, maintaining a low pressure, and must be very smooth to keep the coupling impedance at a level where the beam remains stable. We present a conceptual design of a vacuum system for a high luminosity CESR upgrade using two-in-one quadrupole magnets. The goal of this upgrade is to achieve a luminosity of $3 \times 10^{34} \mathrm{~cm}^{-2} \mathrm{~s}^{-1}$ through a combination of a high beam current $(3.06 \mathrm{~A} / \mathrm{beam})$, low $\beta_{y}$ at the interaction point $(7 \mathrm{~mm})$, and a correspondingly short bunch length. To store the necessary number of bunches, independent vacuum chambers are needed for the two beams. To minimize the size and cost of the storage rings, the dipole magnets are shared between the two rings, and two-in-one quadrupole, sextupole, and steering magnets are used [1,2].

\footnotetext{
* Work supported by the National Science Foundation.

†Email: kwo1@cornell.edu.
}

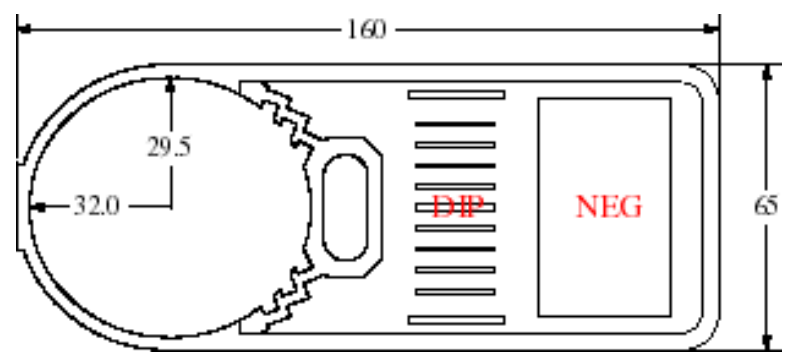

Figure 1: Cross section of the proposed vacuum system design. All dimensions are in millimeters.

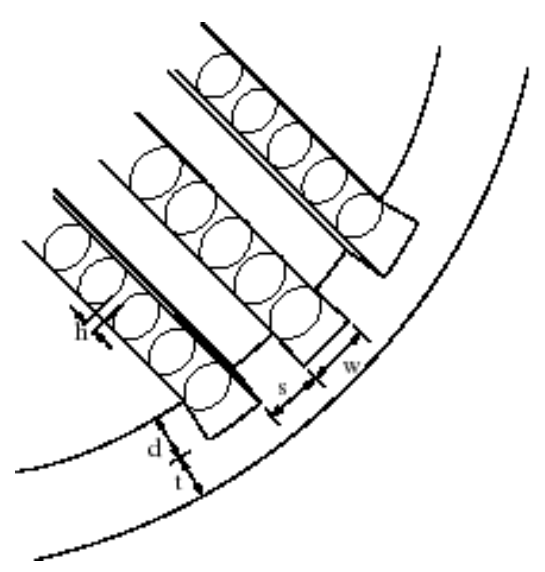

Figure 2: Detail of recessed slots in proposed chamber design.

\section{CHAMBER DESIGN}

The chamber design for the upgrade consists of a copper beam chamber of elliptical cross-section connected to a pumping chamber though holes recessed in long channels in the chamber walls. Figures 1 and 2 show the proposed vacuum system geometry. The parameters we arrived at for our chamber design are shown in Table 1.

Table 1: Vacuum chamber parameters(Refer to Fig. 2)

\begin{tabular}{|lc|c|}
\hline Parameter & & Value \\
\hline Channel width & $w$ & $3.5 \mathrm{~mm}$ \\
\hline Channel depth & $d$ & $2.5 \mathrm{~mm}$ \\
\hline Channel spacing & $s$ & $2 \mathrm{~mm}$ \\
\hline Number of channels & 6 \\
\hline
\end{tabular}




\begin{tabular}{|lc|c|}
\hline Hole radius & $a$ & $1.75 \mathrm{~mm}$ \\
\hline Hole depth & $t$ & $2.5 \mathrm{~mm}$ \\
\hline Hole spacing & $h$ & $1 \mathrm{~mm}$ \\
\hline Total pumping holes per ring & $N_{h}$ & $10^{6}$ \\
\hline
\end{tabular}

\subsection{Impedance}

The inductive part of the impedance of a single hole in a vacuum chamber is given by [3]

$$
\operatorname{Im} Z(\omega)=-\frac{Z_{0} \omega \tilde{e}_{v}^{2}}{2 c}(\psi-\chi),
$$

where $Z_{0}=120 \pi \Omega$ is the impedance of free space, $\tilde{e}_{v}$ is the normalized electrostatic field produced at the hole location by a line charge on the chamber axis, and $\chi$ and $\psi$ are the electric and magnetic polarizabilities of the hole. For a circular beam pipe of radius $b$, the normalized electric field $\tilde{e}_{v}=1 /(2 \pi b)$. The real part of the impedance is given by

$$
\operatorname{Re} Z(\omega)=\frac{Z_{0} \omega^{4} \tilde{e}_{v}^{2}}{12 \pi c^{4}}\left(\psi^{2}+\chi^{2}\right) .
$$

The power per unit length of the beam chamber dissipated due to beam fields scattered by the holes is

$$
P^{\prime}=\frac{N_{h} f_{r e v} q_{b}^{2}}{S_{b}} K
$$

Where $N_{h}$ is the total number of holes, $S_{b}$ is the bunch spacing, $f_{\text {rev }}$ is the revolution frequency and $q_{b}$ is the bunch charge. The loss factor per hole, $K$, is given by

$$
K=\frac{1}{\pi} \int_{0}^{\infty} d \omega \operatorname{Re} Z(\omega) \exp \left[-\left(\frac{\omega \sigma}{c}\right)^{2}\right],
$$

where $\sigma$ is the bunch length.

If the pumping holes are recessed in channels on the walls of the chamber, the normalized field, $\tilde{e}_{v}$, can be reduced by a large factor and thus the impedance would also be reduced [4]. For the current design parameters, the normalized electric field was calculated using POISSON [5]. The electric field at the recessed holes is a factor of 7.5 less than at the wall of the chamber. This gives a factor of 56 reduction in the impedance of the holes.

For the current ring design with recessed holes the loss factor per hole for $7 \mathrm{~mm}$ bunches is $K=4.6 \times 10^{-9} \mathrm{~V}$ $\mathrm{pC}^{-1}$ which corresponds to a power loss of $P^{\prime}=0.68 \mathrm{~W}$ $\mathrm{m}^{-1}$. The total inductive impedance $\operatorname{Im}(Z / n)$ from $10^{6}$ recessed holes is $-2.7 \times 10^{-3} \Omega$, which is a negligible fraction of the expected total impedance of the storage ring.

\subsection{RF Fields}

One of the main reasons for using recessed holes rather than just a few long slots in the chamber is to prevent direct transmission of TE fields generated by the beam into the pumps. The direct beam fields are produced by scattered fields from the holes as calculated above for the loss factor. The power that is directly transmitted to the pumping chamber can be calculated similarly with the inclusion of a factor depending on the cutoff frequency of the holes. The power per unit length transmitted is given by

$$
P^{\prime}=\frac{N_{h} f_{\text {rev }} q_{b}^{2}}{S_{b}} K_{r}
$$

as above in Eq. 3, but where $K_{r}$ includes factors for the cutoff frequency and depth of the holes. For bunch lengths longer than the hole diameter, a long wavelength approximation can be used,

$$
K_{r}=\exp \left(-\frac{2 t \omega_{c}}{c}\right) K
$$

where $\omega_{c}$ is the cutoff frequency of the pumping holes, $t$ is the depth of the holes, and $K$ is the loss factor from before. For the parameters given earlier with $7 \mathrm{~mm}$ bunches, the power radiated through the holes is $P^{\prime}=$ $0.78 \mathrm{~mW} \mathrm{~m}^{-1}$.

\subsection{Conductance}

The size of the holes was limited primarily by the loss factor and RF fields. We calculated the conductance of the holes to determine how many rows of holes would be needed for adequate pumping speed. The conductance of the channels with recessed holes was calculated using MOLFLOW [6]. For the current design parameters the conductance was calculated to be $135 \mathrm{l} \mathrm{s}^{-1} \mathrm{~m}^{-1}$ per channel or $810 \mathrm{l} \mathrm{s}^{-1} \mathrm{~m}^{-1}$ for six channels.

\section{PUMP DESIGN}

The vacuum pumps for the upgrade need to maintain a low chamber pressure when pumping the large gas load due to the increased current in the ring while also being as compact as possible keeping with the compactness of the rest of the design. We settled on a design where the primary pumping will come from distributed nonevaporable getter (NEG) pumps with distributed and lumped ion pumps to supplement for non-getterable gases.

For the NEG pumps, we plan to use standard St707 getter wafer modules from SAES Getters, Inc. These should provide us with the necessary pumping speed in a compact volume. Based on our calculations of the expected gas load, they should need to be reactivated about once a week through heating of the getter strip. 
For the distributed ion (DIP) pumps, we plan on using a plate type pump with only a single row of cells. Table 2 lists parameters for a possible ion pump design. Based on empirical ion pump design formulae [7], we expect a pumping speed of around $85 \mathrm{~s}^{-1} \mathrm{~m}^{-1}$ for our design which should provide us with more than adequate pumping for methane and other non-getterable gases.

Table 2:DIP parameters.

\begin{tabular}{|l|c|}
\hline Parameter & Value \\
\hline Magnetic field strength & $2.0 \mathrm{kG}$ \\
\hline Anode voltage & $5000 \mathrm{~V}$ \\
\hline Cell diameter & $15 \mathrm{~mm}$ \\
\hline Cell height & $39 \mathrm{~mm}$ \\
\hline Anode-cathode gap distance & $5.5 \mathrm{~mm}$ \\
\hline
\end{tabular}

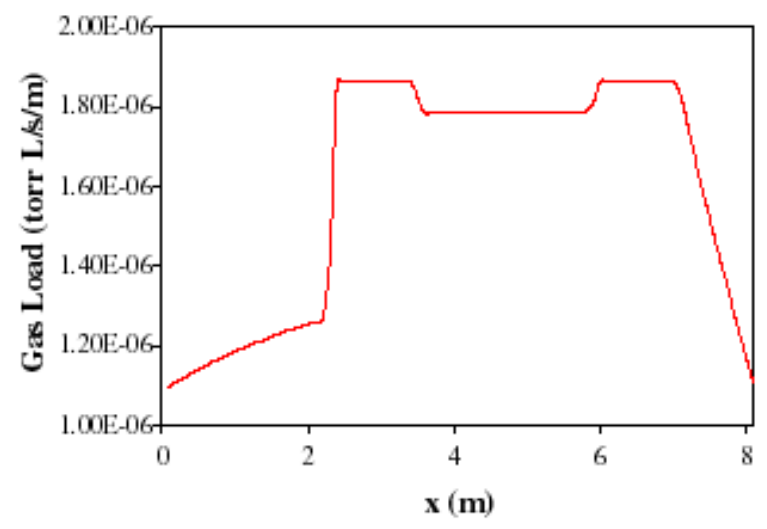

Figure 3: Gas load profile in a half cell of the storage ring arcs.

Figures 3 and 4 show the gas load and pressure profiles in a single half-cell in the storage ring arcs. The regions from 0 to $3.45 \mathrm{~m}$ and 3.55 to $7 \mathrm{~m}$ are within or near the dipole magnets and contain pumping holes and distributed pumps. The region between 7 and $8.1 \mathrm{~m}$ is within or near the quadrupole, sextupole, and steering magnets and does not contain pumping holes. This region is pumped by conduction through the beam pipe. The conductance through the beam pipe is $32.81 \mathrm{~s}^{-1} \mathrm{~m}$. A single $100 \mathrm{~s}^{-1}$ lumped sputter ion pump at $7.1 \mathrm{~m}$ is used to maintain high vacuum when the dipole magnets are turned off. The pressure profile was calculated using a program based on a finite element method [8]. Our program includes in the calculation the conductance of the beampipe and pumping holes as well as allowing for different pumping speeds for NEG and ion pumps and the presence of non-getterable gases. For these calculations we used a DIP pump speed of $85 \mathrm{l} \mathrm{s}^{-1} \mathrm{~m}^{-1}$ and a NEG pump speed of $1000 \mathrm{l} \mathrm{s}^{-1} \mathrm{~m}^{-1}$ was assumed. The photodesorption coefficients used for our calculations are listed in Table 3 [9]. Also listed in Table 3 are the

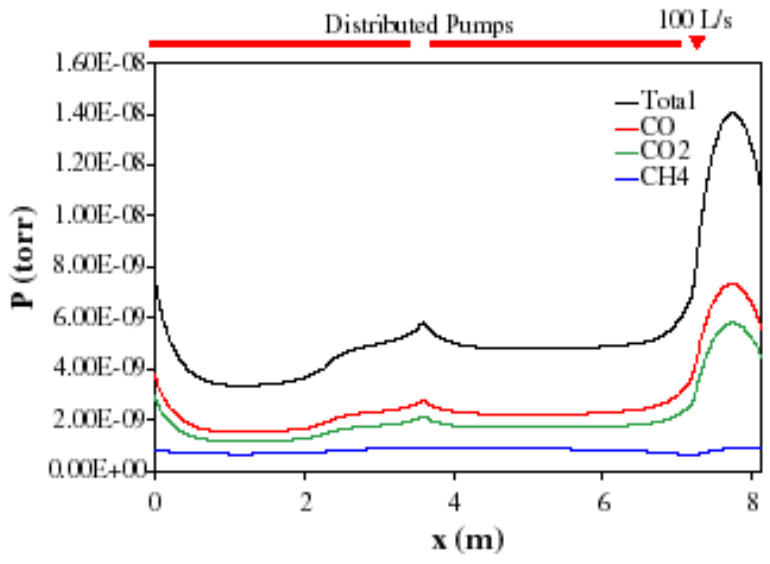

Figure 4: Pressure profile in a half cell of the storage ring arcs.

Table 3: Photodesorption coefficients, partial pressures, and beam-gas lifetimes for different gas species.

\begin{tabular}{|l|c|c|c|}
\hline Gas & $\begin{array}{c}\text { Photodesorption } \\
\text { Coefficient } \\
\text { (mol./photon) }\end{array}$ & $\begin{array}{c}\text { Average } \\
\text { Pressure } \\
\text { (nTorr) }\end{array}$ & $\begin{array}{c}\text { Beam-Gas } \\
\text { Lifetime } \\
\text { (hours) }\end{array}$ \\
\hline $\mathrm{CO}$ & $1.5 \times 10^{-6}$ & 2.7 & 11.3 \\
\hline $\mathrm{CO}_{2}$ & $1.0 \times 10^{-6}$ & 2.1 & 9.1 \\
\hline $\mathrm{CH}_{4}$ & $1.0 \times 10^{-7}$ & 0.8 & 82 \\
\hline \hline Total & & 5.6 & 4.7 \\
\hline
\end{tabular}

results of our calculations for the average pressures and beam-gas lifetimes for the primary gas species we expect. A large pressure bump exists within the unpumped region, but the average total pressure is 5.6 nTorr giving a beam-gas lifetime of 4.7 hours.

\section{CONCLUSION \& FUTURE PLANS}

We have developed a conceptual design of a vacuum system that meets our needs for compactness, low impedance, and low pressure. We are currently in the process of constructing prototype pumps for testing in the near future.

\section{REFERENCES}

[1] A. Mikhailichenko and D. Rubin, Proc. of the 5th European Particle Accelerator Conference (EPAC96), Sitges, Spain (1996) 433.

[2] D. Rubin, G. Dugan, A. Mikhailichenko, J. Rogers, Proc. of the 1997 Particle Accelerator Conference (PAC97), Vancouver, BC, Canada (1997) 318.

[3] S.S. Kurennoy and Y.H. Chin, Part. Accel. 52, 201 (1996).

[4] T. Weiland, Part. Accel. 51, 53 (1995).

[5] Los Alamos Accelerator Code Group, "The POISSON/SUPERFISH Group of Codes", LANL report LA-UR-87-115 (1987).

[6] R. Kersevan, "MOLFLOW User's Guide", Sincrotrone Trieste Technical Report, ST/M-91/17 (1991).

[7] Y. Suetsugu and M. Nakagawa, Vacuum 42, 761 (1991).

[8] Y. Li, Cornell LNS Report CBN 97-7 (1997).

[9] C.L. Foerster, et. al., BNL report BNL-48367, 1992. 